\title{
Usefulness of spinal unenhanced computed tomography and CT-myelography in the age of multidetector CT technology and magnetic resonance imaging - Preliminary considerations
}

\author{
Mario Ricciardi ${ }^{1, *}$, Angela Campanella ${ }^{2}$, Gloria Grieco ${ }^{3}$ and Roberta Zammit ${ }^{3}$ \\ ${ }^{1}$ Pingry Veterinary Hospital, Via Medaglie d'Oro 5, 70126 - Bari, Italy \\ ${ }^{2}$ Ospedale Veterinario Gregorio VII. Piazza di Villa Carpegna 52, 00165 - Roma, Italy \\ ${ }^{3}$ Clinica Veterinaria Borghesiana, Via di Vermicino 96, 00133 - Roma, Italy
}

\begin{abstract}
Over the last decade, magnetic resonance imaging (MRI) and multidetector computed tomography (MDCT) have revolutionized diagnostic potential in small animal practice, providing adequate assessment of spinal diseases at levels comparable to that achieved in human radiology. T2-weighted MRI images are extremely sensitive to intramedullary parenchymal disorders, while balanced steady-state free precession sequences provide high-quality myelographic images of the spine without the need of intrathecal contrast medium administration. Multidetector computed tomography, with its near-isotropic spatial resolution and multiplanar reformatting of the acquired datasets, provides sufficient stratigraphic details of the spinal cord and the epidural space, facilitating the detection of compressive pathologies without the need of subarachnoid opacification. Nowadays, MDCT and low-field (LF) MRI have become fairly standard and available in academic institutions and private veterinary facilities, appearing to be valuable, complementary, and non-invasive diagnostic tools for imaging the spine. In this scenario, this clinical communication provides a series of preliminary observations that may help to reconsider the usefulness of CT-myelography in the light of its invasiveness and actual diagnostic advantages compared to MRI and unenhanced MDCT for the assessment of compressive and non-compressive spinal diseases in small animals.
\end{abstract}

Keywords: Cat, Computed tomographic myelography, Dog, Multidetector computed tomography, Spinal cord.

\section{Introduction}

Spinal neuromusculoskeletal disorders are relatively common in small animal practice and include a wide range of different entities, which, independently from their etiopathogenetic mechanism, may lead to different dramatic clinical presentations, from spinal pain to severe limb dysfunction (Lorenz et al., 2011; Platt and Garosi, 2012). In patients with signs of spinal cord dysfunction, advanced imaging evaluation of the spine is essential after survey radiographs for many pathologic conditions and may be obtained with computed tomography (CT), computed tomographic myelography (CTM), and magnetic resonance imaging (MRI). Each imaging technique has different properties in term of contrast and spatial resolution, which in turn affect the final diagnostic detail for the main different spinal components, such as spinal cord, intervertebral discs, vertebrae, and paravertebral soft tissue. Regardless of the technique adopted, all advanced imaging evaluations in spinal patients aim, basically, to detect: 1) compressive spinal cord pathologies and 2) parenchymal spinal cord lesions. The first group includes traumatic injuries (vertebral fracture and luxation), compressive disc diseases (Hansen type I disc extrusion, Hansen type II disc protrusion, and hydrated nucleus pulposus extrusions), extramedullary hemorrhage, extramedullary neoplasms, epidural inflammations and anomalies (e.g. arachnoid diverticula, congenital compressive vertebral stenosis/malformations). The second group includes vascular diseases (e.g. ischemic myelopathy), inflammatory conditions (e.g. infectious/non-infectious meningomyelitis), infiltrative parenchymal neoplasms, and non-compressive disc disease (acute noncompressive nucleus pulposus extrusions - ANNPE) (Jeffery et al., 2013; Hague et al., 2015; Wisner and Zwingenberger, 2015; Ricciardi, 2016; Mauler et al., 2017).

Multidetector computed tomography (MDCT) is the most recent breakthrough of CT technology, deriving from the evolution of the older axial and single-slice spiral CT scanners, and is widely available among veterinary facilities (Ricciardi, 2016). The advantages of this technique for spinal imaging in small animal practice derive from scan speed, large anatomical coverage, and near-isotropic spatial resolution with excellent anatomical detail for vertebral and muscular components without superimposition. In addition, MDCT provides good morphological detail for spinal cord tissue and epidural fat, but low contrast resolution 
in the evaluation of mild intraparenchymal attenuation changes (Ricciardi, 2016).

Computed tomographic myelography (CTM) is a modification of the standard CT technique, in which the images are acquired after the subarachnoid administration of non-ionic iodinated contrast medium via cisternal or lumbar puncture. The aim of this procedure is to obtain opacification of the subarachnoid space in order to enhance the distinction between the spinal cord, the arachnoid layer, and the epidural space (de Lahunta and Glass, 2009) and to identify any deviations or attenuations that may reflect the site of a spinal cord compression or presence of intradural expansive lesions (Robertson and Thrall, 2011) (Fig. 1). Since the spinal cord has been traditionally considered not clearly assessable on CT images (Olby et al., 2000), CTM is classically conceived to increase the visualization of compressive spinal cord pathology, enhancing the identification of non-mineralized extradural and intradural extramedullary lesions. These indications include disc herniation, neoplasia, hematoma, granuloma/abscess, cyst (arachnoid or synovial), scar tissue, and empyema (da Costa and Samii, 2010). In parallel with the evolution of CT technology, since the 1990s, MRI has revolutionized the diagnostic abilities in small animal practice, providing adequate assessment of neurologic diseases, almost comparable to that achieved in human radiology. Low-field (LF) scanners are common in veterinary facilities, providing excellent diagnostic images of the spine. Two-dimensional (2D) Spin echo (SE) sequences are the mainstay of a standard LF-MRI protocol in conjunction with inversion recovery (IR) sequences and, in the majority of conditions, they are sufficient to characterize structural abnormalities or parenchymal signal changes of the spinal cord (Dennis, 2011; Robertson and Thrall, 2011). In particular, T2weighted SE and balanced steady-state free precession (bSSFP) sequences, with their bright CSF signals, provide high-quality myelographic images of the spine without the need of intrathecal contrast medium administration (Ricciardi, 2018). Overall, the most relevant inconvenience encountered in small animal MRI, compared to MDCT, is the low spatial resolution of images, which, in turn, affects their anatomic detail. However, MDCT image quality is limited by a low sensitivity in the detection of intraparenchymal changes (i.e. intramedullary spinal cord lesions). In this scenario, in which MDCT and MRI appear to be valuable, complementary diagnostic tools for imaging the spine, this preliminary clinical communication analyses, through a series of case examples and a brief review of the literature, the current usefulness of unenhanced CT and CTM in the evaluation of compressive and non-compressive spinal cord diseases in small animals.
Identifying compressive spinal cord pathologies with different CT technologies

While it is well accepted that most intraparenchymal lesions of the spinal cord are not or barely identifiable using X-ray-based imaging modalities, such as radiographs and $\mathrm{CT}$, a continuous effort has been pursued so far in veterinary radiology to detect compressive extramedullary pathologies. For many years, since the development of non-ionic, watersoluble, iodinated contrast media, myelography was the principal imaging modality used to delineate the spinal cord and to visualize, with multiple radiographic projections, the sites and sides of compressions (Olby et al., 2000; Robertson and Thrall, 2011; Ricciardi, 2016).

When CT machines have become commonly available in academic institutions and private veterinary facilities, the procedure of subarachnoid opacification has been combined with CT imaging of the spine, giving rise to CTM, which eliminates the problem of superimposition that affects radiographs, thus enhancing the myelographic assessment. The assumption at the bases of employment of CTM is that any non-mineralized extramedullary lesion reaching an attenuation value almost comparable to the spinal cord parenchyma may be not or difficult to be distinguished, and the degree of spinal cord compression could be underestimated. Here, CTM, enhancing the contrast resolution between the spinal cord edge (the perimedullary arachnoid space) and the epidural space, has been intended to solve this problem. Before 1998, CT technology was largely based on Computed Axial Tomography (CAT) and single-slice spiral machines (Bertolini and Prokop, 2011). Such machines have a great limitation in spatial resolution, essentially linked to the large slice thickness value required to cover large anatomic regions per each acquisition. With thick slices (4-5 mm), volume averaging can reduce lesion conspicuity (Robertson and Thrall, 2011), especially when large spinal segments are examined. Furthermore, the limitation in spatial resolution directly influences the reformattability of the acquired dataset, making sagittal and dorsal images unsuitable for an accurate spinal evaluation.

Hence, the evaluation of the spinal cord only in the transverse plane, at large thickness values, made the identification of small extramedullary compressive lesions difficult and largely entrusted to the great differences in the attenuation value between the lesion and spinal cord section (e.g. mineralized epidural disc herniation). In this context, CTM was useful and facilitated the detection of compressive lesions. With the advent of MDCT machines, the spatial resolution of the acquired dataset reached almost the isotropy, with great multiplanar reformattability of the images in multiple spatial planes. This technique has 
revolutionized the CT imaging of the spine, which, extending over a longitudinal axis, is optimally analyzed in the sagittal and dorsal planes.

Moreover, the near-isotropic spatial resolution and multiplanar image analysis of MDCT greatly improved the achieved morphologic and stratigraphic detail of the spinal cord and the epidural space. With modern highresolution MDCT datasets, the low Hounsfield attenuation value of the epidural fat surrounding the spinal cord produces myelographic-like images, especially on dorsal MPR visualization, comparable to those achieved with CTM and MRI, which allows a non-invasive evaluation of compressive spinal cord disorders (Dennis, 2011) (Fig. 2 and 3). The myelographic-like effect provided by the epidural fat is particularly evident at the level of thoracic and lumbar spine were the extramedullary adipose tissue is more aboundant, and less evident - but still appreciable - in the cervical spinal segment, especially in toy breed dogs and cats (Fig 2E, 2F).

Usefulness of CT- myelography in the evaluation of extramedullary spinal cord diseases

\section{Extramedullary spinal cord hemorrhage}

Computed tomography can identify acute hemorrhage in the vertebral canal, without the need of subarachnoid contrast medium injection (Olby et al., 2000). In dogs, reported causes of extraparenchymal spinal cord hemorrhage include coagulopathies, brown snake envenomation, haemophilia, hemorrhage associated with neoplasia, trauma, spinal surgery, angiostrongylosis and spontaneous (idiopathic) hemorrhages (Wessmann et al., 2006; Hague et al., 2015).

However, the most commonly reported CT pattern of extramedullary spinal hemorrhage is that associated with acute disc herniation due to damage of vertebral venous sinuses (Olby et al., 2000; Griffin et al., 2009). Epidural hemorrhage typically appears slightly more attenuating than the spinal cord and may be found as a single hyperattenuating mass, mixed with disc material at the site of disc herniation or as a narrow ring-like rim of hyperattenuation around the spinal cord sections near or distant to the primary site of disc herniation. In the latter case, it is believed to represent epidural blood spill (Olby et al., 2000; Griffin et al., 2009).

Interestingly, CTM has been considered not useful in the characterization of epidural hemorrhage, since vertebral sinus hemorrhage can result in a filling defect that extends over several vertebral bodies and cannot be differentiated from an extensive extradural compression or spinal cord swelling (Brisson, 2010).

In the authors' experience, primary spinal hemorrhage has been observed also in a case of angiostrongylosis infestation, in which an extensive hyperattenuating extramedullary space-occupying lesion was seen dorsal to the spinal cord, in the lumbar segment, and as ring- like attenuation change around the thoracic spinal cord. In this case, the unenhanced CT findings matched the MRI findings, with precise identification of a localization of a presumptive extramedullary hemorrhage (Fig. 4).

Compressive disc diseases (Hansen type I disc extrusion, Hansen type II disc protrusion, and hydrated nucleus pulposus extrusions)

Intervertebral disc herniation (IVDH) is a common cause of spinal cord compression in dogs and a typical indication for spinal imaging (Robertson and Thrall, 2011). Basically, the imaging evaluation in the case of disc herniation aims to assess: 1) the location and extension of the herniated disc material, 2) the laterality, 3) the degree of spinal cord compression, and 4) the presence and extension of compressive myelopathy in patients with loss of deep pain perception (DPP).

All this information facilitates an eventual surgical treatment and orients the prognosis (Robertson and Thrall, 2011; Ricciardi, 2016). Except for cases requiring MRI to evaluate spinal cord parenchymal damage (compressive myelopathy) associated with disc herniation, dogs with preserved DPP may be adequately evaluated by CT of the spine, which is considered an accurate imaging modality for the assessment of disc herniations (Olby et al., 2000; Hecht et al., 2009; Israel et al., 2009; Dennison et al., 2010).

\section{Hansen type I disc protrusions}

So far, the main drawback in the diagnosis of Hansen type I IVDH using plain CT has been that nonmineralized extruded material, or small amounts of poorly mineralized material, could be difficult to detect (Jeffery et al., 2013). Today, this assumption can be reconsidered. In a study on the CT appearance of acute disc herniation in $23 \mathrm{dogs}$, the presence of herniated disc material in the vertebral canal was apparent without the injection of sub-arachnoid or intravenous contrast medium, even in animals where little disc material was herniated.

Both mineralized and non-mineralized disc materials were clearly visible in the epidural space. Mineralized disc material, associated with epidural hemorrhage, appeared only slightly more attenuating than the spinal cord, but was clearly distinguishable from the nervous tissue and extended over distances of up to five vertebrae (Olby et al., 2000). In another study on 43 dogs with disc herniation, the location of displaced nonmineralized disk material was correctly identified by loss of epidural fat or displacement of the spinal cord. Furthermore, no significant differences between noncontrast CT and MRI were found, highlighting that canine displaced disc material can be seen in CT images without the need for CT-myelography (Harder et al., 2015). This is confirmed by the authors' experience (Fig. 5 and 6). 


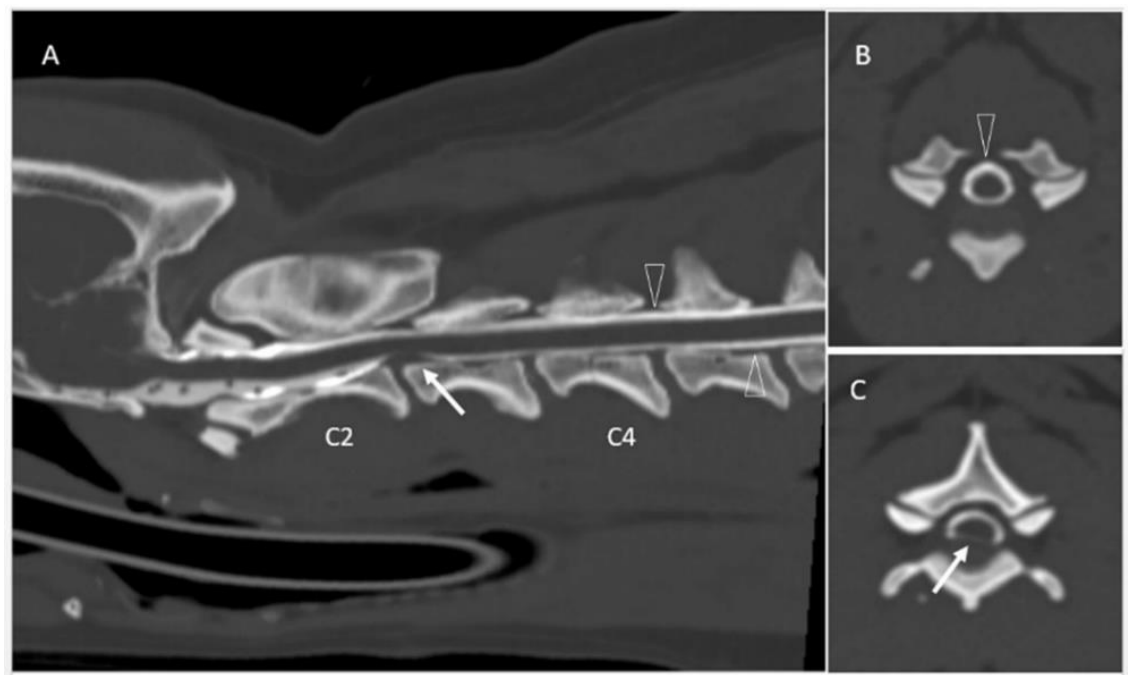

Fig. 1. (A): Midsagittal computed tomographic myelography of the cervical spine in a 5-year-old beagle dog with C2-C3 disc protrusion (arrow). Note the perimedullary enhancement of the arachnoid layer obtained after cisternal injection of iodinate contrast medium (arrowheads). (B): Transverse image at the level of C4-C5 intervertebral disc space showing a ring-like appearance of the enhancing CSF layer around a normal spinal cord section. (C): Transverse image at the level of the C2-C3 intervertebral disc space, showing deviation of the ventral CSF layer secondary to the disc compression (arrow).

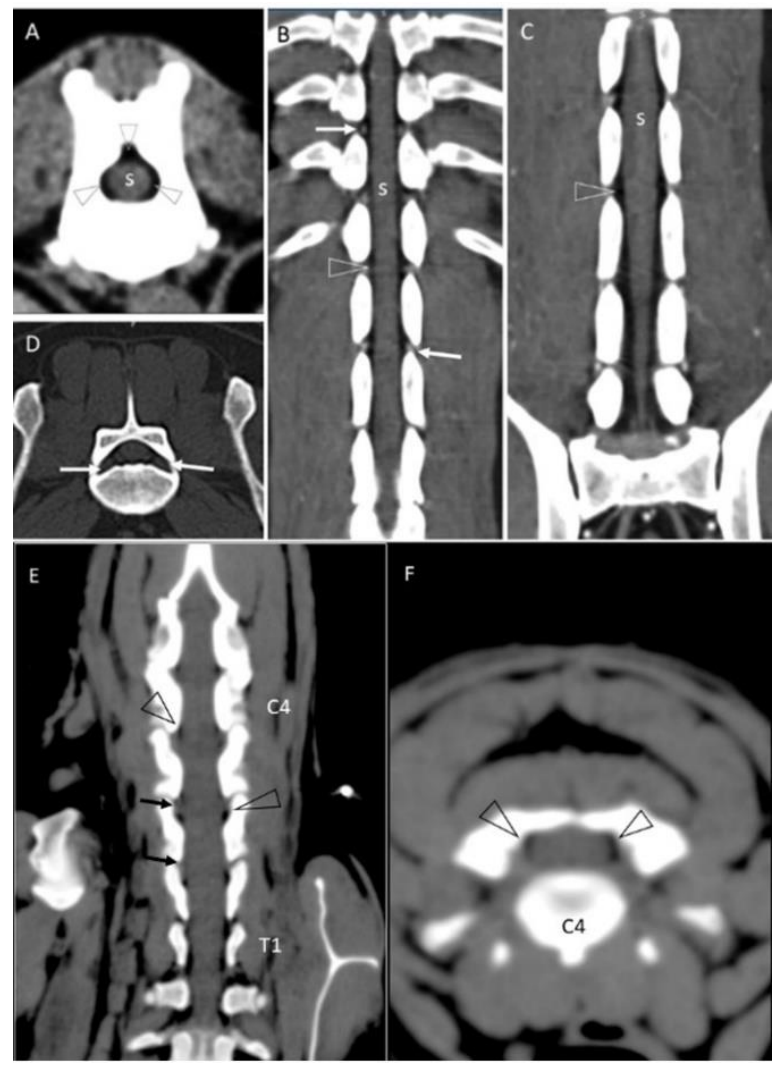

Fig. 2. Normal MDCT images of the spine in a 6-year-old mixed breed dog. (A): transverse image at the level of T12; (B): dorsal MPR image at the level of the thoracolumbar segment; (C): dorsal MPR image at the level of the lumbosacral segment; (D): transverse MPR image at the level of L7. The near-isotropic spatial resolution of the MDCT dataset provides excellent stratigraphic details of the spinal cord (s) and the epidural space, with a natural myelographic-like effect deriving from the perimedullary hypoattenuating epidural fat (arrowheads). Note the detailed visualization of the nerve root at the level of neuroforamina (arrows). (E): Dorsal and (F): transverse image (at the level of C4) of the cervical spinal cord in a in a 7-year-old dachshund. The myelographic-like effect provided by the epidural fat (arrowheads) is less aboundant - but still appreciable - in the cervical spinal segment, especially in toy breed dogs and cats. 


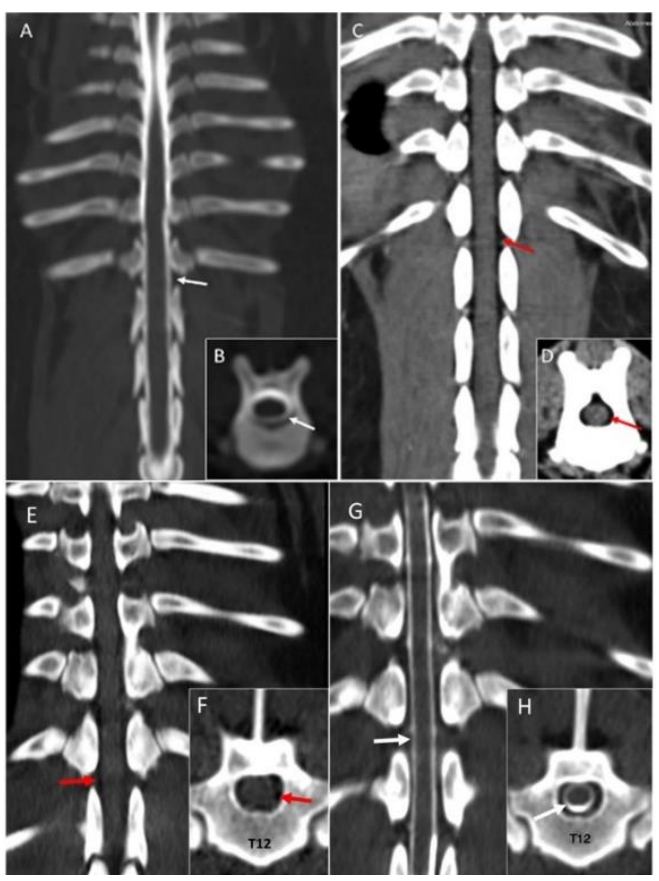

Fig. 3. Comparison between myelographic effect of the computed tomographic myelography (CTM) and unenhanced MDCT. (A): Dorsal computed tomography myelography in a 5-year-old female pug at the level of the thoracolumbar segment and (B): transverse image at the level of T12. (C): Dorsal MPR unenhanced MDCT image of the toracolumbar segment in a 6-year-old mixed breed dog and (D): transverse image at the level of T12. Comparison between unenhanced MDCT (E, F): and CTM (G,H): in a 6 yearold male Corso dog - thoracolumbar segment. On CTM images, the spinal cord silhouette is outlined by the contrast medium administered in the subarachnoid space (A, B, G, H - white arrows). On unenhanced MDCT images, a myelographic-like effect is naturally provided by the perimedullary epidural fat (C, D, E, F - red arrows). The high contrast and spatial resolution of MDCT provides myelographic-like images in a non-invasive way. Images E-H are courtesy of Dr. Tatiana Venuti (Clinica Veterinaria Aretusia, Siracusa (SR), Italy).

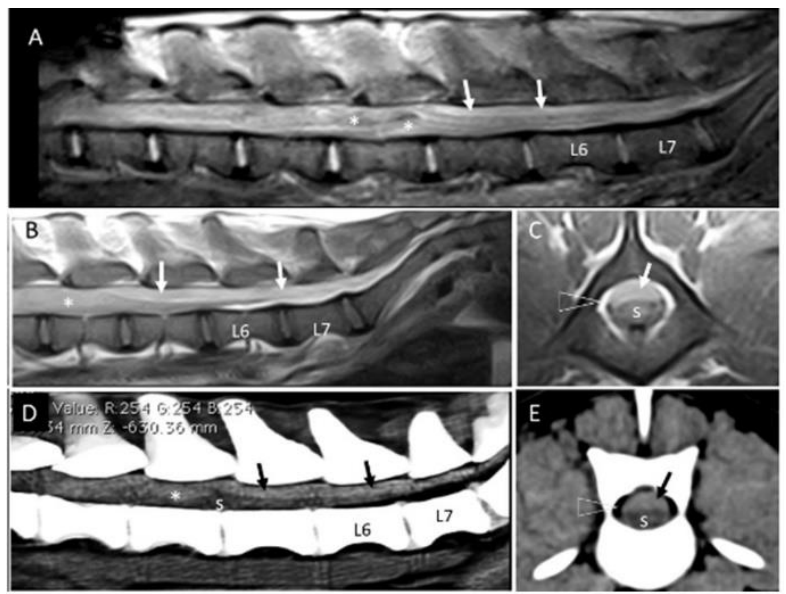

Fig. 4. Unenhanced MRI and MDCT images of the lumbosacral spinal cord in an 8-month old female mongrel dog with intraduralextramendullary hemorrhage due to angiostrogylosis. (A): Midsagittal gradient-echo MRI image showing hyperintense dorsal extramedullary linear pathologic material in the L5-L7 spinal segment (arrows) and intraparenchymal hypointensity (asterisk). (B): Midsagittal ad (C): transverse - at L5 level - T1-weighted spin-echo MRI images showing diffuse hyperintensity of both intra- and extramedullary components of the lesion. Overall, the signal behavior of the lesion in both spin-echo and gradient-echo images is suggestive of intra- and extramedullary hemorrhage. (D): Midsagittal ad (E): transverse - at L5 level - MDCT images showing diffuse hyperattenuation of both intra- (asterisk) and extramedullary (black arrows) components of the lesion. From the comparison of MRI and MDCT images, there is no significant difference in the visualization of the intra- and extramedullary hemorrhage. Note that in both MRI and MDCT transverse images (C, E), the pathologic extramedullary material is seen under the epidural fat layer (arrowheads) and compresses the spinal cord (s). Also, in the MDCT images, note the great attenuation difference between the spinal cord parenchyma and the extramedullary hemorrhage, which allows accurate assessment of the lesion without the need of CTM. 


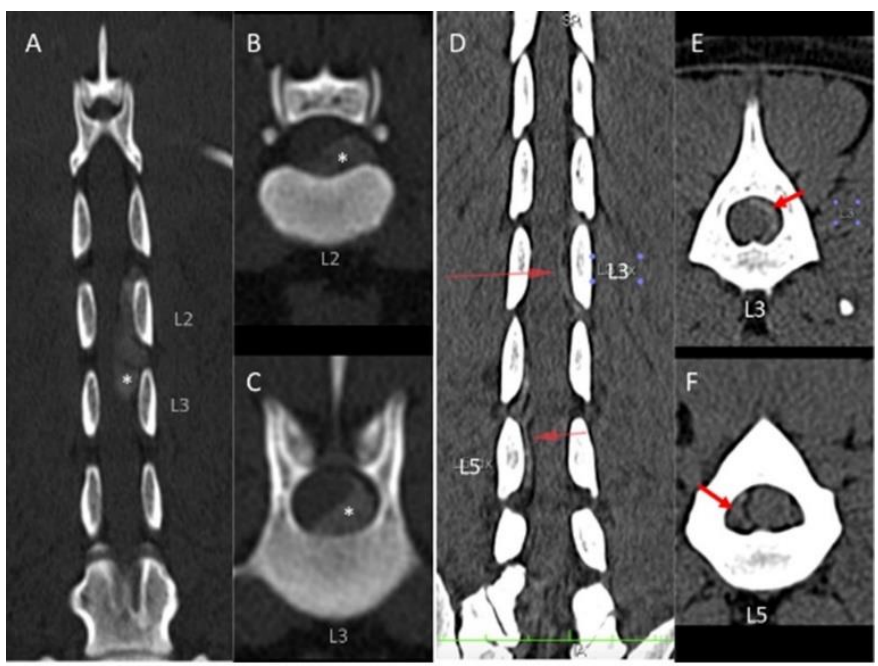

Fig. 5. Unenhanced dorsal (A): and transverse (B, C): MPR MDCT images of the lumbar spine in a 3-year-old poodle dog with Hansen type I disc extrusion at the L2-L3 intervertebral disc space with left lateralization. Note the difference in the attenuation value between the partially mineralized extruded disc material (asterisks) and the spinal cord. Unenhanced dorsal (D): and transverse (E,F) MPR MDCT images of the lumbar spine in a 5-year-old French bulldog with Hansen type I disc extrusion. The epidural extruded disc material is displaced medially to the left vertebral lamina of L3 and from the L4-L5 to the L5-L6 intervertebral disc space on the right. Note that the compressive material is barely hyperattenuating (E): or isoattenuating (F): to the spinal cord (suggesting low mineralization), but it is clearly visible on unenhanced CT images as it occupies the space of the normal epidural fat (loss of hypoattenuating extramedullary layer at the level of the spinal cord compression). During decompressive surgery, extruded disc material mixed to the hemorrhagic component was removed.

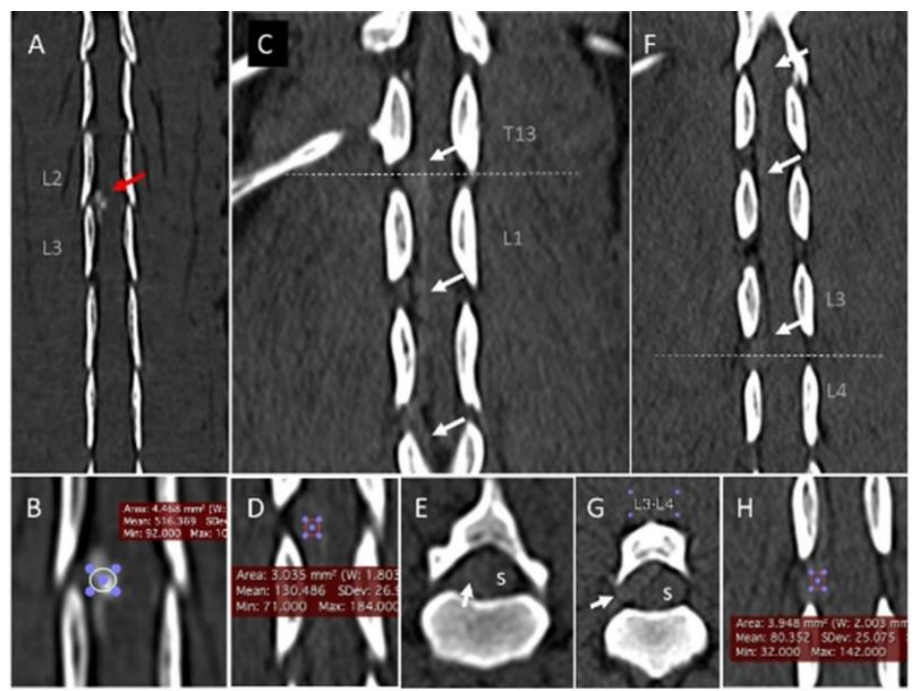

Fig. 6. (A): Unenhanced dorsal MPR MDCT image of the lumbar spine in a 13-year-old DSH cat with strongly mineralized Hansen type I disc extrusion at L2-L3 intervertebral disc space with right lateralization (red arrow). (B): Detail of the mineralized disc material with high mean attenuation value (516 Hounsfield units). (C): Unenhanced dorsal MPR MDCT image of the lumbar spine in a 7-year-old mongrel dog with less mineralized Hansen type I disc extrusion at the T13-L1 intervertebral disc space with right lateralization. Note that the extramedullary compressive material extends caudally beyond the site of herniation. (D): Detail of the extruded disc material with a mean attenuation value of 130 Hounsfield units, suggesting mild mineralization or large amounts of hemorrhagic components. (E): Transverse image at the level of the dotted line in image C. Note the compressive material (white arrows) still distinguishable from the spinal cord ( $\mathrm{s}$ ) at any level, even in the absence of high mineralization (C, E). (F): Unenhanced dorsal MPR image of the lumbar spine in the same dog. The compressive material (white arrows) extends caudally, reaching the L3-L4 disc space. At this level, it shows very low mineralization, appearing almost isoattenuating to the spinal cord. (G): Transverse image at the level of the dotted line in image F, showing the contrast between the compressive disc material (arrow) in the right dorsolateral position and the spinal cord (s). (H): Detail of the extruded disc material with a mean attenuation value of 80 Hounsfield units, suggesting very low mineralization or large amounts of hemorrhagic components. Note that the extramedullary compressive material is identifiable in the epidural space, regardless of the degree of mineralization. 
Interestingly, in human medical literature, the diagnostic accuracy of plain CT, CTM, and MRI has been compared for the diagnosis of nerve compression caused by lumbar disc extrusions in 95 patients with low-back pain. Results indicated no statistically significant difference in the diagnostic accuracy of the three modalities for the diagnosis of disc-related nerve root compression (Thornbury et al., 1993)

\section{Hansen type II disc protrusions}

The diagnostic sensitivity of plain MDCT in nonmineralized chronic disc protrusion (Hansen type II) is currently unknown and has not been compared with the sensitivity of CTM (da Costa and Samii, 2010).

In a study on forty-six dogs with compressive spinal cord disease, CT demonstrated low sensitivity for nonmineralized Hansen type II disc protrusions in comparison to CTM (Dennison et al., 2010). However, such data were obtained from CT images acquired with a single-slice helical CT scanner. Today, modern MDCT scanners produce high quality images with high contrast and spatial resolution, allowing detailed identification of disc protrusion and adequate distinction between spinal cord section and hypertrophied annulus fibrosus, irrespective of its mineralization (Fig. 7).

\section{Compressive hydrated nucleus pulposus extrusions}

Hydrated nucleus pulposus extrusions (HNPE), previously misinterpreted as discal cysts (Konar et al., 2008; Kamishina et al., 2010), are a type of disc herniation in which non-degenerated or partially degenerated extruded disc material distributes within the fibers of the dorsal longitudinal ligament or in the vertebral canal, causing spinal cord compression (Dolera et al., 2015). Magnetic resonance imaging features are characteristic and show nuclear material of hydrated signal intensity immediately dorsal to the affected disc space, often with a "seagull" shape on transverse T2-weighted images (Beltran et al., 2012; Dolera et al., 2015; Manunta et al., 2015). Although MRI is the imaging modality of choice for such type of disc herniation, in a study on 11 dogs with cervical HNPE, the compressive disc material was visible in all patients in contrast-enhanced CT (intravenous administration of iodinated contrast media). The hydrated extruded disc component appeared hypoattenuating to the spinal cord, with rim enhancement and often associated with narrowing of the intervertebral disc space. In this study, the sensitivity for detection of a lesion consistent with HNPE on CE-CT was $91 \%$, with a specificity of $100 \%$ (Royaux et al., 2016) (Fig. 8).

Infectious and noninfectious inflammatory spinal cord disorders

Inflammatory spinal cord diseases are commonly reported in small animal practice and include intraparenchymal infiltrative lesions and space- occupying masses of both infectious and noninfectious origin (Wisner and Zwingenberger, 2015). Advanced imaging may orient the clinical suspicion, but diagnosis of such disorders is often made from signalment, clinical presentation, cerebrospinal fluid (CSF) analysis, and response to therapy (Wisner and Zwingenberger, 2015).

The two most common noninfectious inflammatory spinal diseases associated with visible imaging findings in dogs are granulomatous meningoencephalomyelitis (GME) and spinal idiopathic sterile pyogranulomatous inflammation (ISPI). Descriptions of CT features of spinal GME are lacking in veterinary medical literature, but they would be expected to be subtle or absent (Wisner and Zwingenberger, 2015). Being in the majority of cases an intraparenchymal infiltrative disease of the spinal cord, GME is not apparent on CT images due to the low contrast resolution for soft tissue of this imaging modality in comparison to MRI (Cooper et al., 2014; Ricciardi, 2016), only rarely, slight patchy intramedullary enhancement may be seen after intravenous contrast medium administration (Fig. 9). CT myelography is unlikely to provide adjunctive details in cases of intramedullary GME with preservation of the spinal cord silhouette, except for cases in which severe focal parenchymal swelling can attenuate the subarachnoid enhancement. Such a finding, however, is not specific and could not replace CSF examination for the final diagnosis of GME.

In contrast to GME, ISPI is characterized by epidural inflammatory aggregation. In the early stage of cell aggregation, a modification of normal epidural fat attenuation value is seen, making the lesion readily apparent around the spinal cord. As long as the cell aggregate increases its volume, the granulomatous lesion becomes a truly compressive epidural neoformation and displaces the spinal cord. Idiopathic sterile pyogranulomatous inflammation has been reported in a dog using plain MDCT. The lesion appeared as an epidural expansive mass in the thoracolumbar spinal segment, hypoattenuating to the soft tissue (CT value of $-38 \mathrm{HU}$, suggestive of prevalent adipose component) without contrast enhancement (adipose granuloma) (Murata et al., 2012). In the authors' experience, non-infectious epidural granulomatous lesions may also appear as extramedullary masses isoattenuating to the soft tissue with no contrast enhancement (Fig. 10). The latter possible appearance makes such lesions more easily detectable on the epidural fat background than adipose granuloma. In both early and advanced stages, the inflammatory tissue may be recognized within the epidural space on plain MDCT, and CTM probably would not provide any adjunctive information. As for any inflammatory disorder of the CNS, further characterization should rely on CSF examination. 


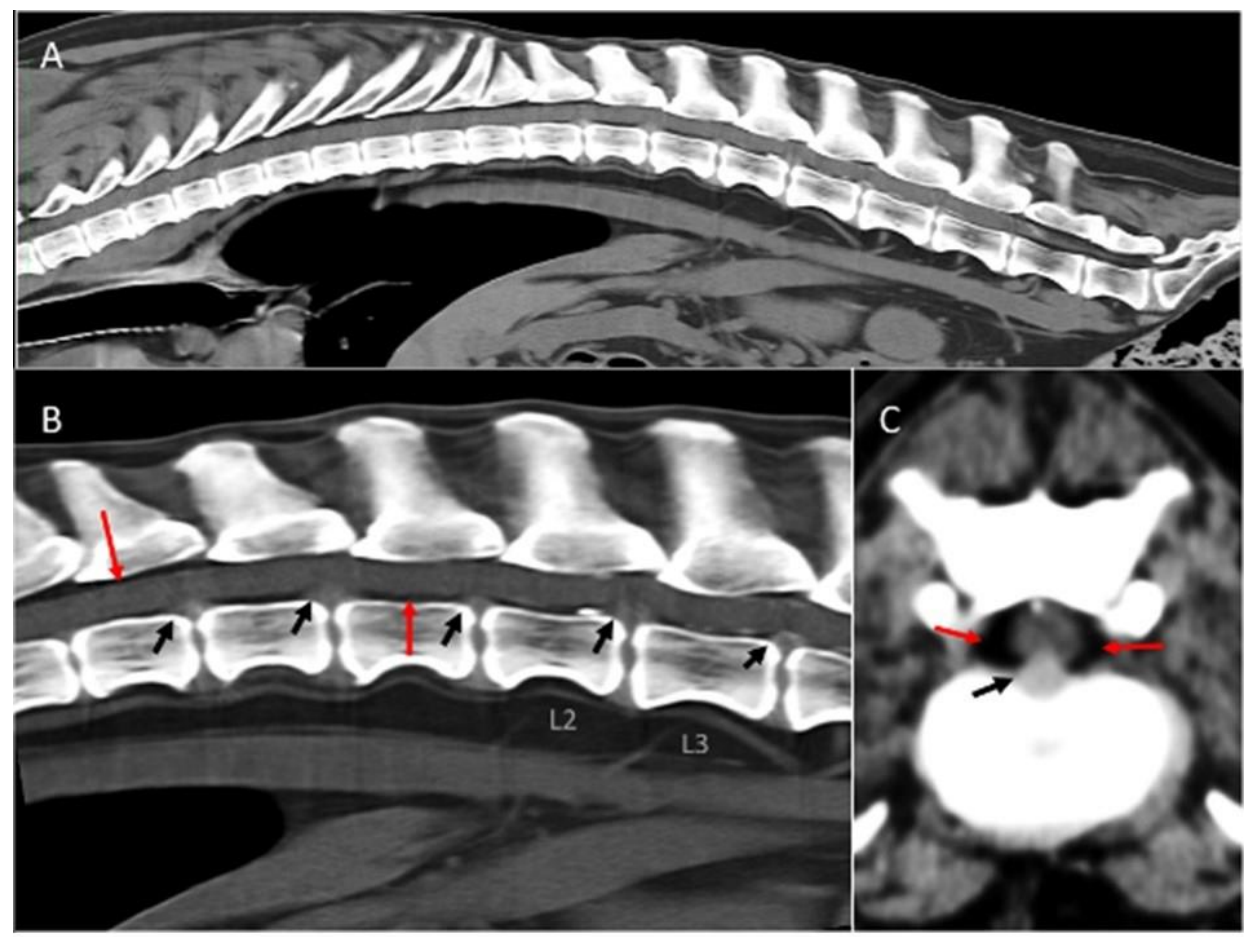

Fig. 7. (A): Midsagittal MPR MDCT image of the thoracolumbar spine in a 7-year-old female German shepherd with multiple non-mineralized Hansen type II disc herniations. (B): Detail of the most affected spinal segment (T11-L4). Note the myelographic effect provided by the epidural fat (red arrows), interrupted at the compression sites (black arrows). (C): Transverse MDCT image at the level of the L2-L3 intervertebral space. Note the difference between the protruded annulus fibrosus (black arrow) and the spinal cord. Also note the perimedullary epidural fat (red arrows).
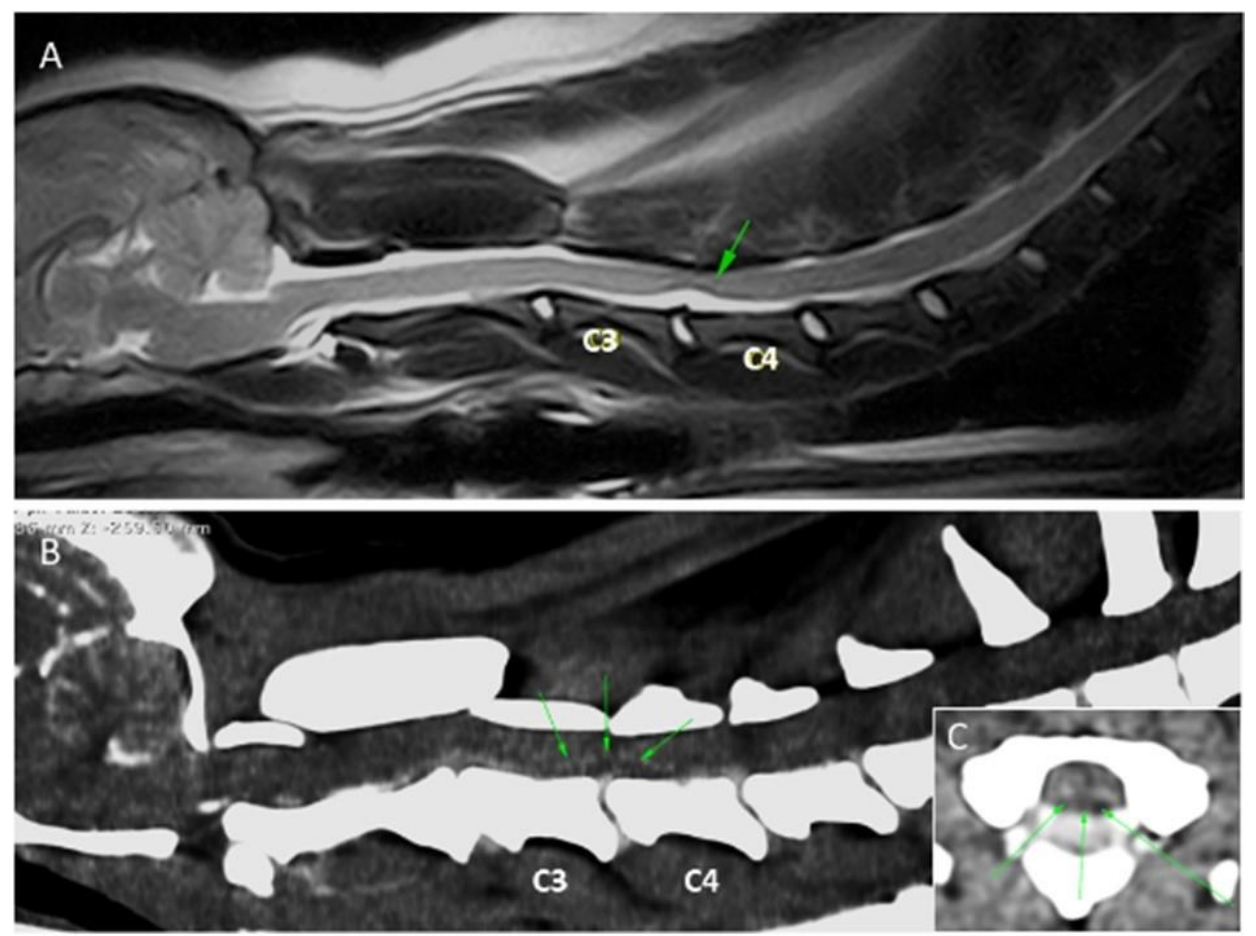

Fig. 8. (A): Midsagittal T2-weighted MRI image of the cervical spine in a 5-year old male mixed-breed dog with C3-C4 hydrated nucleus pulposus extrusions (arrow). (B): Midsagittal and (C): transverse MDCT images after intravenous contrast medium administration in a 6-year-old male beagle dog with C3-C4 hydrated nucleus pulposus extrusions (arrows). On CT images, the hydrated compressive disc material appears hypoattenuating compared to the spinal cord, with mild rim enhancement (arrows). 

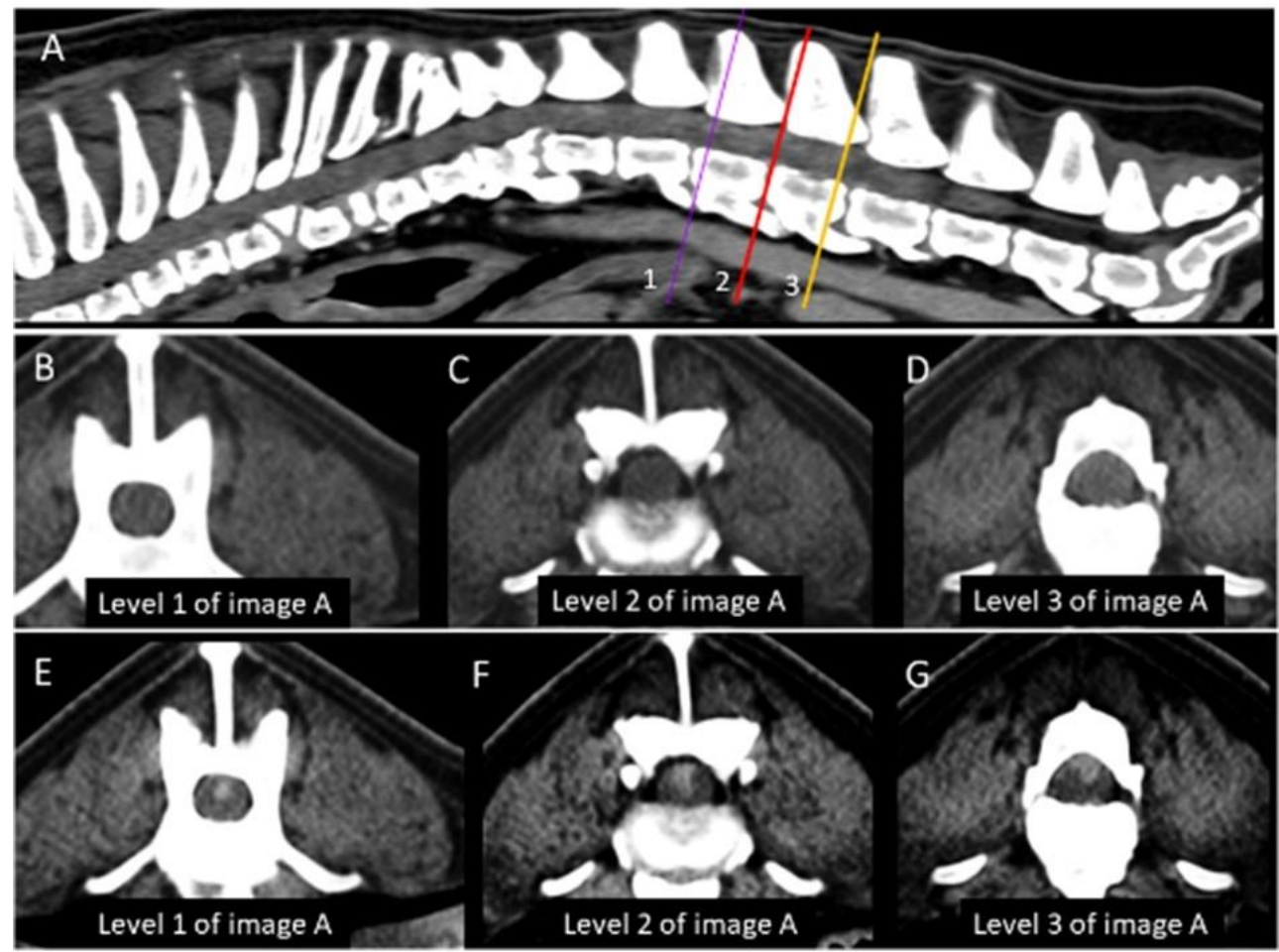

Fig. 9. (A): Midsagittal MDCT image after intravenous contrast medium administration of the thoracolumbar spine in a 6-year-old male French bulldog with granulomatous meningoencephalomyelitis and prevalent intramedullary infiltration. (B, C, D): Transverse unenhanced images at level 1, 2, 3 of image A, respectively. (E, F, G): Transverse images at level 1, 2, 3 of image A, respectively, after intravenous contrast medium administration. Note the patchy intramedullary enhancement within the spinal cord section after intravenous contrast medium administration.

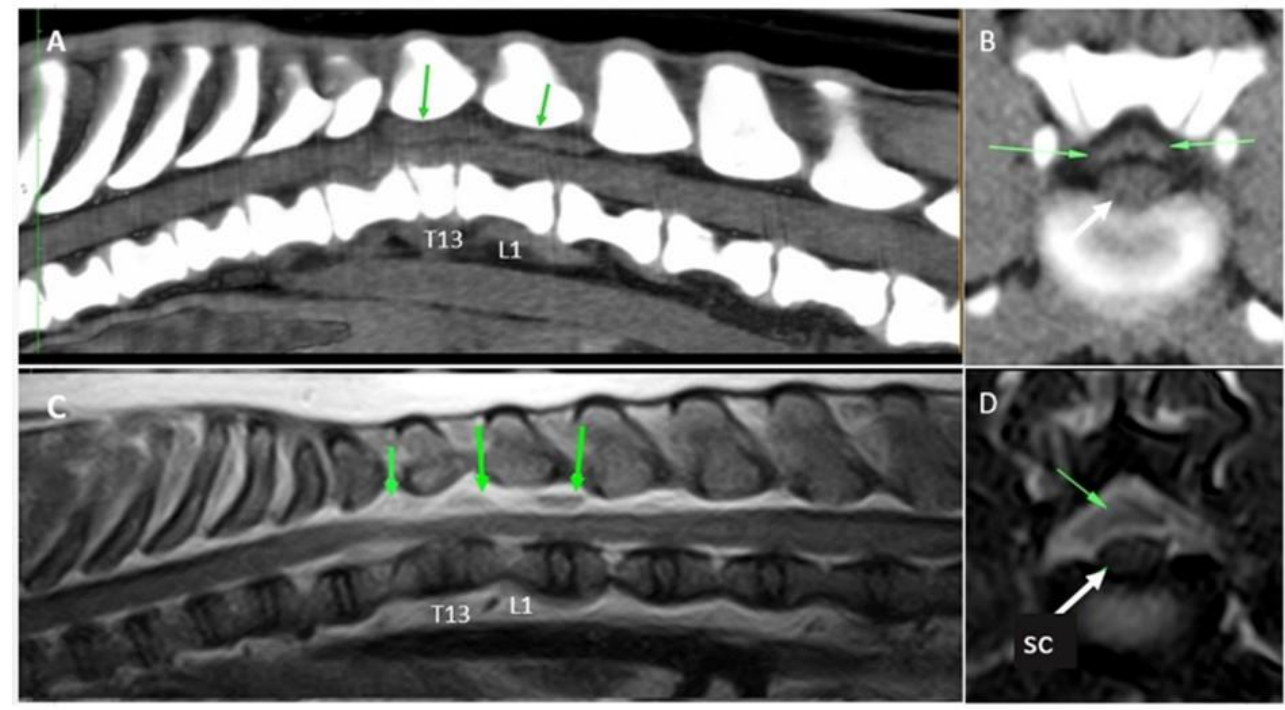

Fig. 10. (A): Midsagittal and (B): transverse (at the level of L1) unenhanced MDCT images of the thoracolumbar spine in a 1-yearold female English bulldog with idiopathic sterile pyogranulomatous inflammation. There is an evident attenuation change of the dorsal epidural fat in the T13-L1 segment, resembling an extramedullary space-occupying mass (green arrows). (C): Midsagittal and (D): transverse T1-weighted spin-echo MRI images of the same dog at the same levels as in images A and B. There is an evident signal change of the dorsal epidural fat in the T13-L1 segment (green arrows). No significant difference is seen between MRI and unenhanced MDCT images for the characterization of the epidural fat inflammation. White arrows in image B and D point to the spinal cord sections (sc). 
Primary infectious extramedullary space-occupying lesions (epidural empyema and infectious granuloma) are rare in small animals and generally derive by direct extension of discospondylitis, other local infection, or through hematogenous dissemination (Wisner and Zwingenberger, 2015). The few available descriptions of such conditions in small animals are based only on MRI imaging (Cauduro et al., 2011; Favole et al., 2013; Reginato et al., 2014); however, a case of epidural empyema of the cervical spine, evaluated through CT and CTM, has been reported for a dog. In this case, the unenhanced CT scan demonstrated a soft-tissue mass at the level of the C3-4 intervertebral disk space, which resulted in the loss of the normal low attenuating epidural fat prior to CTM (Nykamp et al., 2003).

\section{Extramedullary neoplasms}

As any expansive extramedullary lesion, spinal cord neoplasm interrupts the epidural fat layer and displaces the spinal cord, so that they become apparent on plain MDCT images (Fig. 11). To date, a comparison between CTM and plain MDCT for the identification and characterization of the spinal cord neoplasia is lacking in veterinary medical literature. The superiority of CTM over plain CT in distinguishing between epidural and intradural neoplasm has not been proven so far in small animal practice, so that the presumptive advantages of reaching a more accurate pre-surgical characterization of the tumor's origin should be weighted against the risk of subarachnoid contrast administration.

Spinal cord and vertebral anomalies (arachnoid diverticula; vertebral stenosis/malformations)

Spinal arachnoid diverticula (SAD) are found in the cervical and thoracic spine, most commonly as focal dilations of the dorsal or dorso-lateral CSF layer, and may exert a clinically detectable compressive effect on the spinal cord (Mauler et al., 2017). Through selective contrast-enhancement of the subarachnoid space, CTM provides high details of SADs, showing focal accumulation of the contrast medium, starting gradually and ending abruptly with a "tear-drop" enlargement of the CSF layer on sagittal and transverse MPR images. No associated intramedullary lesion (compressive myelopathy) is detectable via CTM examination (Bismuth et al., 2014) (Fig. 12A). When evaluated on MRI, SADs may be less easily identified on T2-weighted spin echo sequences, which return almost equally bright signals from both the CSF and the surrounding epidural fat and from adjacent intraparenchymal compressive myelopathy. Furthermore, in LF-MRI scanners the slice thickness cannot be set below $3 \mathrm{~mm}$ on T2-weighted SE sequences, providing insufficient spatial resolution for delineation of SAD, both in sagittal and transverse images. Due to these spatial and contrast resolution limits, parenchymal and dilated CSF (SAD) compartments may be indistinguishable on T2weighted spin echo sequences, especially in small breed dogs with small spinal cord diameter (i.e. the boundaries between CSF, epidural fat, and compressive myelopathy are not clearly delineated) (Seiler et al., 2012). However, in the last years, with the evolution of MRI technology, new two- and three-dimensional sequences have been developed, which provides more detailed myelographic images comparable to those achievable with CTM. Half-Fourier acquisition singleshot turbo spin-echo (HASTE) sequences are available with high-field systems, and when obtained with a long echo time (TE), tissues with a relatively long T2relaxation time, such as CSF, return high signal intensity, whereas other tissues, including fat, have no or low signals. The detection of arachnoid diverticula increased significantly with the availability of a HASTE sequence in a standard spinal MRI sequence protocol (Seiler et al., 2012). bSSFP is a highresolution, volumetric, bright-fluid 3D-Gradient Echo sequence, provided among standard equipment in LFMRI scanners. If acquired in the sagittal plane, bSSFP provides optimal myelographic images of the spine, highlighting only the CSF layer and diverticula and reducing, with its unique image contrast, any misleading (not useful) spinal cord hyperintensity: the CSF dilation and its boundaries are clearly differentiated from the compressive myelopathy (Ricciardi, 2018) (Fig. 12B). Nowadays, the wide availability of MRI scanners among veterinary facilities should prompt clinicians to choose, among CTM and MRI, the highest myelographic detail with the less invasive imaging modality in patients with suspected SADs. Compressive congenital disorders of the spine most commonly derive from vertebral malformation (hemivertebrae, butterfly vertebra, atlanto-axial malformations, cervical spondylomyelopathy) (Bailey, 1975; Morgan, 1999; Newitt et al., 2008) or inherited degenerative joint disease of the articular processes in young dogs (McDonnell et al., 2003). Such developmental disorders of the axial skeleton, commonly detected in brachycephalic and toy breeds of dogs, either individually or in combination, may lead to stenosis of the vertebral canal (Berlanda et al., 2011). In such conditions, an unenhanced CT scan allows adequate assessment of the type of vertebral anomaly, the degree of spinal canal stenosis or spinal cord compression (Berlanda et al., 2011). As for traumatic vertebral injuries, this is possible because the near-isotropic spatial resolution of the MDCT offers good morphological details for the compressed spinal cord segment(both in the transverse and sagittal plane), in the case of severe stenosis, and for the reduction/loss of epidural fat in the case of subtle narrowing of the spinal canal (Ricciardi, 2016) (Fig. 13). 

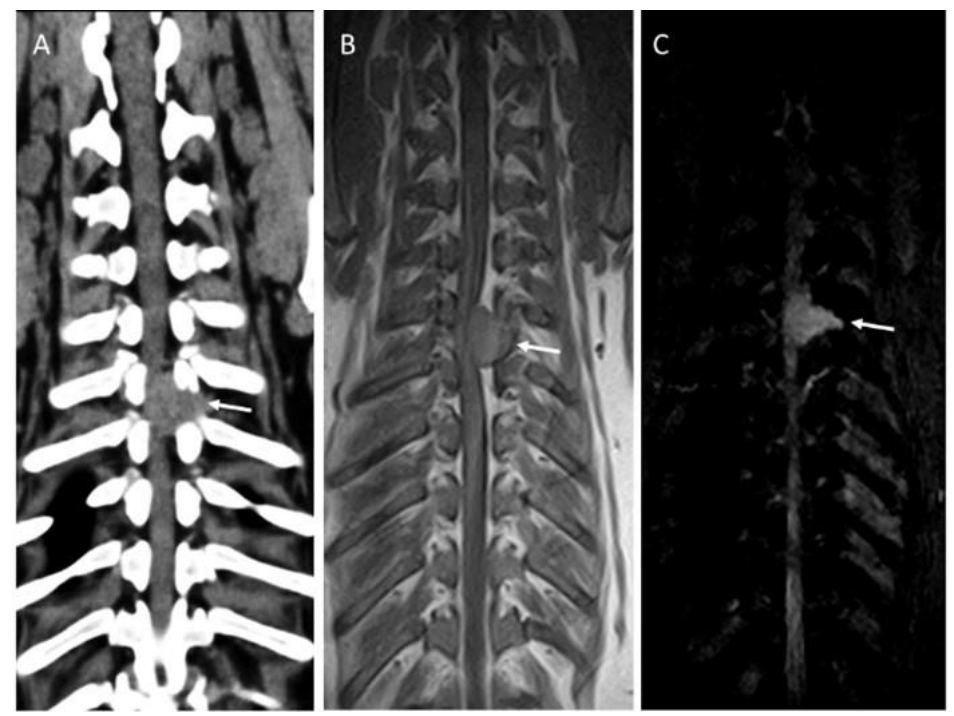

Fig. 11. (A): Unenhanced dorsal MPR MDCT, (B): T1-weighted spin-echo and (C): fast STIR MRI images of the thoracic spine in a 13-year-old male mixed-breed dog with an intradural extramedullary hemangioblastoma at the level of the T5-T6 intervertebral space (arrows). The extramedullary expansion of the neoplasm leads to the loss of the epidural fat layer on the CT image. Furthermore, the slight difference in attenuation between the mass and the spinal cord allows differentiation of the tumor. Note the invasion of the T5-T6 intervertebral neuroforamina. There is no significant difference in the morphologic assessment of the extramedullary neoplasm between MDCT and MRI images.

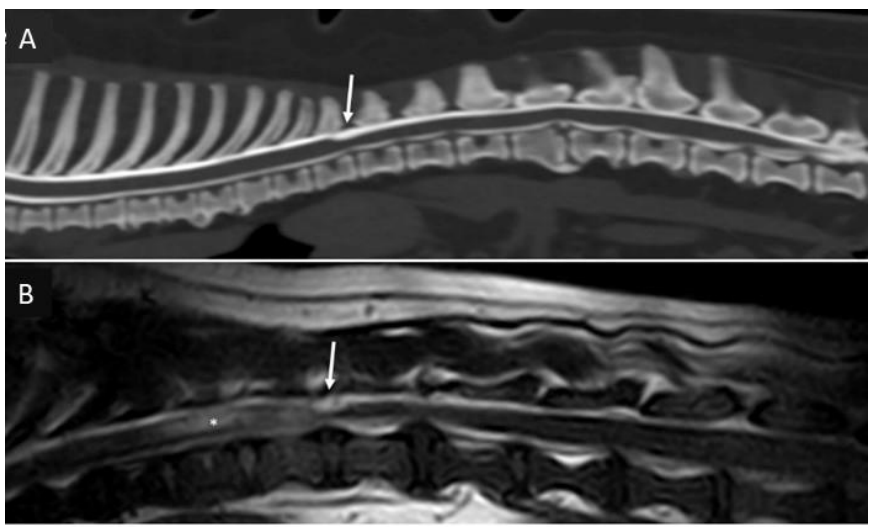

Fig. 12. (A): Midsagittal computed tomography myelography in a 5-year-old female pug with T10-T11 dorsal arachnoid diverticulum (arrow). (B): Left parasagittal multiplanar reformatted bssfp MRI image in a 10-year-old male pug with left dorsolateral T12-T13 arachnoid diverticulum (arrow). MRI provides optimal morphologic details of the arachnoid layer, without the need of subarachnoid contrast medium administration. Moreover, MRI images permit the assessment of the presence and extension of compressive myelopathy, seen as intramedullary hyperintensity on bright-fluid sequences (B, asterisk), which is of important prognostic value. CTM does not provide information on parenchymal attenuation changes secondary to spinal cord compression.

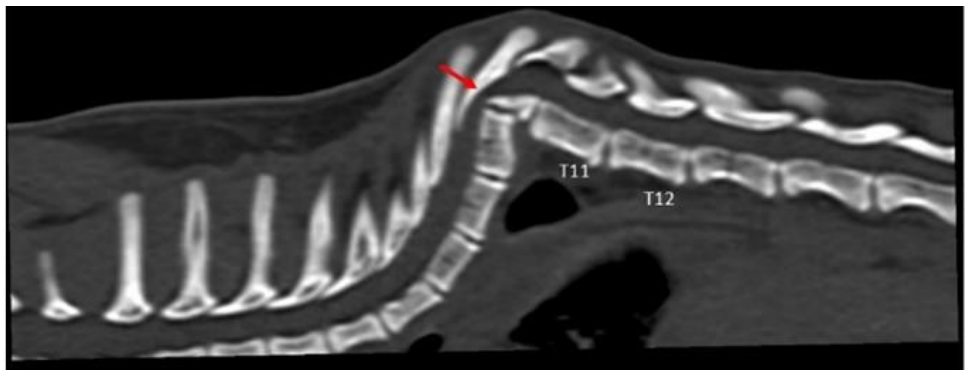

Fig. 13. Midsagittal MPR unenhanced MDCT image in a 5-month-old domestic shorthair cat with malformation of T10 (wedgeshaped vertebral body), T9-T10 malarticulation, and stenosis of the vertebral canal. The spinal cord silhouette and its narrowing at the level of the vertebral stenosis (arrow) is clearly assessable. 


\section{Traumatic injuries (vertebral fracture and luxation)} In the case of traumatic vertebral injuries, CTM has been described as useful in defining the presence and extent of spinal cord compression due to fracture displacement (Wisner and Zwingenberger, 2015). However, this assumption may be questionable, since the inherent high contrast resolution of CT for the skeletal component allows for the detection of the narrowing of the vertebral canal or the presence therein of fracture fragments, without the need of intravenous or subarachnoid contrast medium administration (Ricciardi, 2016).

In a review on the usefulness of multidetector $\mathrm{CT}$ in the evaluation of spinal traumatic disorders in small animals, CTM has been described as not useful in the evaluation of compressive vertebral injuries because of the scarcity of additional information achievable, the required positioning which is potentially detrimental for a traumatized vertebral column, and the invasive nature of such a technique (Ricciardi, 2016). Furthermore, the presence of contrast medium in the subarachnoid space may obscure small bone fragments within the spinal canal (Jeffery, 2010). Moreover, plain CT allows for concurrent evaluation of extradural compression by traumatic hemorrhage, which may appear hyperattenuating to the spinal cord parenchyma (Shores and Brisson, 2017). If the blood in the epidural space shows attenuation comparable to the spinal cord parenchyma, it can be detected by evaluation of epidural fat loss.

Usefulness of CT- myelography in the evaluation of intraparenchymal spinal cord diseases

Intraparenchymal non-compressive spinal cord diseases commonly diagnosed in small animal neuroimaging include: ischemic myelopathy, infectious/non-infectious myelitis, intramedullary neoplasms, and acute non-compressive nucleus pulposus extrusions (ANNPE) (De Risio et al., 2009; Masciarelli et al., 2017). Except for solid, expansive intramedullary neoplasms, all these diseases may not be associated with a significant spinal cord enlargement and changes of the spinal cord silhouette (Masciarelli et al., 2017). This implies that, in such cases, the subarachnoid space is not compressed or deviated and CTM results in a normal myelographic image. In ANNPE, the reduction of intervertebral disc space in the site of herniation on CT images may indirectly orientate the diagnostic suspicion in the absence of evident epidural compressive disc material and in association with clinical findings (Ricciardi, 2016). However, for inflammatory intraparenchymal infiltrates, pure infiltrative neoplasm, or ischemic damages, a normal CTM may miss the imaging diagnosis (Cooper et al., 2014).

Intramedullary solid expansive neoplasms or infiltrative tumors, associated with severe parenchymal swelling, may be suspected in CTM images by the attenuation of arachnoid enhancement cranially and caudally to the epicenter of spinal cord enlargement (Lorenz et al., 2011).

However, even in such cases, if the tumor is not associated with significant enhancement after intravenous contrast medium administration, CT and CTM may fail to identify the boundaries of the lesion against the background of normal spinal cord tissue. Intramedullary tumors may exhibit variable or even absent contrast enhancement (Masciarelli et al., 2017) so that in both contrast-enhanced CT (intravenous contrast medium administration) and CTM, their location and extension with respect to vertebral landmarks may be poorly assessed.

This assumption is applicable also to intramedullary granulomatous meningoencephalomyelitis, which has also been reported to show no enhancement after intravenous contrast media administration (Fisher, 2002; Cherubini et al., 2006). On the other hand, intramedullary neoplasms and inflammatory lesions that show enhancement after intravenous contrast media administration can be seen and characterized without the need of CTM (Fig. 9).

Hence, since intramedullary spinal cord lesions are often challenging to characterize based only on CT/CTM images and biopsy; especially for those that do not contact the pial surface, MRI is considered to be a key test for making diagnoses in the clinical setting, along with CSF analysis (Masciarelli et al., 2017).

\section{Disadvantages of CT-myelography}

There are several widely described side effects and disadvantages related to the procedure of myelography/CT-myelography, basically deriving from the invasiveness of the technique:

- Technical difficulty of the test, especially for lumbar myelography compared to cisternal injection, which requires a high level of skill to perform it safely and without excessive prolongation of the imaging and anesthetic procedure (Paithanpagare et al., 2008; Robertson and Thrall, 2011).

- Risk of incorrect injection site. For cisternal myelography, subdural injection, with the spreading of contrast medium between the dura mater and the arachnoid membrane, is the most common complication observed. This leads to an artefactual appearance of the spinal cord silhouette (undulating border on the inner aspect of the dorsal contrast layer), which may preclude a diagnosis or lead to an erroneous conclusion (Penderis et al., 1999). A similar alteration in the spinal cord silhouette may be seen in the case of epidural spreading of the contrast medium, which is considered a more frequent inconvenience of lumbar injection (Paithanpagare et al., 2008). However, spontaneous leptomeningeal leakage of contrast medium with epidural distribution 
may also occur as a result of increased permeability secondary to traumatic, ischemic, or inflammatory meningeal disease (Carstens and Kitshoff, 2007). Central canal injection of contrast medium has also been reported (Kirberger and Wrigley, 1993). These complications arise from the incorrect positioning of the needle or movement of the dog during injection, especially if the depth of the anesthesia is insufficient.

- Myelography-induced seizures. In veterinary practice, the most commonly reported complications from myelography are seizures (Barone et al., 2002; da Costa et al., 2011). Second-generation non-ionic contrast agents (iopamidol, iohexol, iotrolan, and iomeprol) are routinely used for myelography/CTmyelography in dogs. Among these, iohexol, the most used agent, has been associated with focal or generalized post-myelographic seizures due to a chemically induced meningitis, with a reported frequency ranging from 3 to $21.4 \%$. Although CTM requires lower doses of contrast medium than conventional myelography, large breed dogs having large volumes $(>8 \mathrm{~mL})$ of iohexol injected into the cerebellomedullary cistern had the highest risk of seizures (da Costa et al., 2011).

- Iatrogenic trauma. Penetration of the brainstem and spinal cord may occur for cisternal or lumbar myelography. Intramedullary injection of contrast medium has been associated to severe parenchymal damage such as spinal cord edema, parenchymal necrosis, myelomalacia, and hydromelia (Kishimoto, et al., 2003; Funkquist, 2008) (Fig. 14).

- Cardiac arrhythmias and hemorrhage within the cord or vertebral canal have also been reported, even after correct administration of the contrast agent into the subarachnoid space (Parker et al., 1975; Carroll et al., 1997). Fatal diffuse subarachnoid and leptomeningeal hemorrhage throughout the entire length of the spinal cord, brain stem, and brain base has been also reported as a potential complication of lumbar myelography in dogs (Packer et al., 2007).

- Insensitivity of CT for intraparenchymal disease. One of the most useful advantages of MRI in the imaging setting of compressive spinal cord disease is the possibility to visualize and assess the extension of associated compressive myelopathy (CM). In severe compressive disease, $\mathrm{CM}$ is seen on T2-weighted sequences as intramedullary linear hyperintensity, which extends cranially and caudally from the site of spinal cord compression. The evaluation of its presence end extension provides important prognostic information in patients with loss of deep pain perception, especially for the surgical outcome (probability of a full functional recovery) and for the risk of developing ascending-descending myelomalacia (ADMM) (Ito et al., 2005; Balducci et al., 2017). Computed tomography provides low contrast resolution in the evaluation of intraparenchymal attenuation changes, and intraparenchymal modification secondary to compression of the spinal cord may not be apparent (Cooper et al., 2014; Ricciardi, 2016).

- Long duration of anesthesia. Minimizing the time under anesthesia is desirable: the risk of systemic hypotension is higher under general anesthesia; hypotension can reduce blood flow to the injured spinal cord and worsen the outcome (Tator and Fehlings, 1991).

\section{Conclusion}

Magnetic resonance imaging (MRI) has been traditionally considered as the procedure of choice for spinal neuroimaging due to fewer potential side effects with no radiation exposure or subarachnoid contrast injections and the superior tissue imaging detail obtained.

Nowadays, MDCT technology can offer equal or superior details for structural/compressive spinal cord pathology, providing myelographic-like images in a non-invasive way and faster scan time. For the majority of compressive epidural disorders, the loss of epidural fat layer may be, in general, sufficient to localize the lesion and to characterize its extension on unenhanced MDCT images.

For subtle space-occupying lesions with attenuation values similar to that of the spinal cord, intravenous contrast media administration may enhance visualization. For intramedullary spinal cord lesions and arachnoid diverticula, CTM does not provide superior information in comparison to MRI and does not permit the assessment of compressive myelopathy. In the author's daily practice, MDCT scans of the spine - both native and, if necessary, after intravenous contrast medium administration - constitute the primary imaging modalities for dogs suspected of an acute compressive myelopathy, since they are faster, less expensive than low-field MRI, and provide all the required diagnostic information to orient surgical planning in patients with intact DPP. In cases of spinal lesions not apparent on first plain MDCT evaluation, MRI and CSF evaluation could be preferred over CTM, since they provide more diagnostic information without the risks associated with subarachnoid contrast medium administration.

The retrospective nature of this study and the small number of case examples shown, limit the strength of any conclusions that can be made. However, the evidence provided by these preliminary observations suggests that today the usefulness of CTM may be reconsidered. Nevertheless, further research is needed to determine the overall statistical significance of the CTM compared to unenhanced MDCT and MRI on detecting compressive and non-compressive spinal diseases in small animals. 


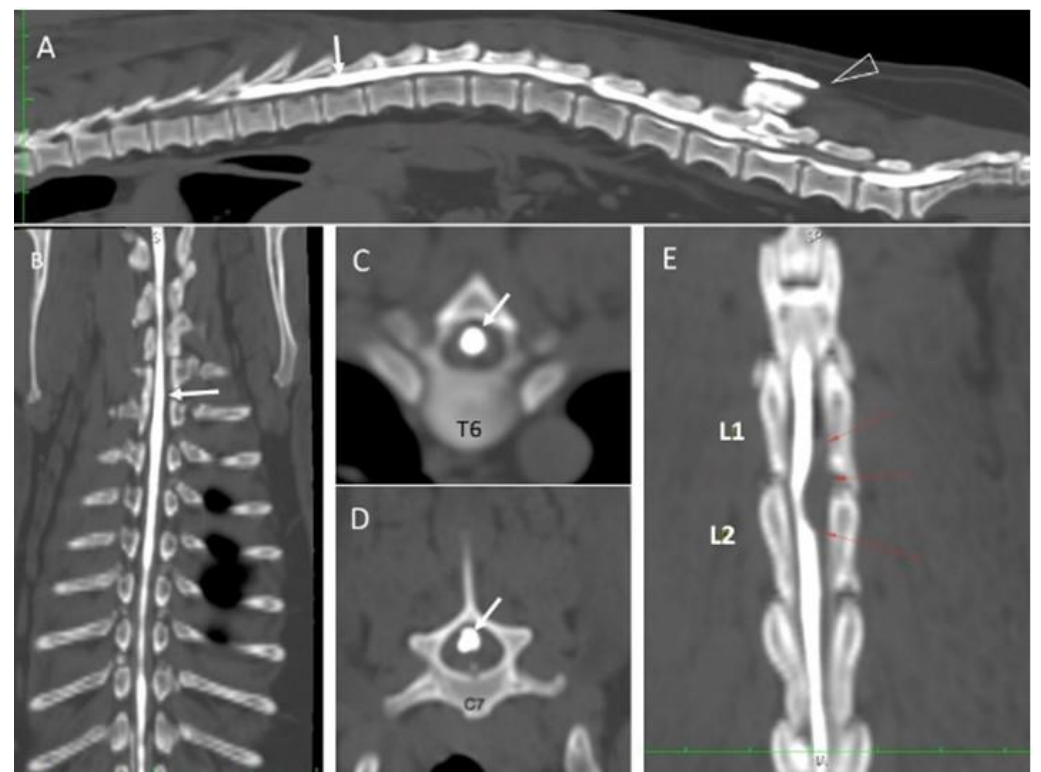

Fig. 14. Three-year-old male dachshund with acute paraparesis due to a presumptive left-sided Hansen type I disc extrusion at the L1-L1 intervertebral space. The dog was referred for worsening of the neurologic condition subsequent to a lumbar computed tomography myelography (CTM). CTM procedure lead to intramedullary linear enhancement from the site of injection (arrowhead) to the cervical spinal cord seen on sagittal (A) and dorsal (B) multiplanar reformatted images. On transverse images, the contrast medium was seen in the center of the spinal cord sections instead of as a perimedullary ring (C, D arrows). The compressive extramedullary lesion is seen at the L1-L1 intervertebral space, displacing the intramedullary column of contrast medium (E, red arrows). Intramedullary injection of contrast medium is one of the possible inconveniences of the CTM and has been associated with severe iatrogenic parenchymal damage.

\section{Acknowledgments}

The authors wishes to thank Dr. Gilberto Pollina (Clinica Veterinaria Borghesiana, 00133 Rome, Italy), Dr. Tatiana Venuti (Clinica Veterinaria Aretusia, Siracusa (SR), Italy) and the staff of the Pingry Veterinary Hospital of Bari (Italy) for their assistance with the data collection.

\section{Conflict of interest}

The Authors declare that there is no conflict of interest.

\section{References}

Bailey, C.S. 1975. An embryological approach to the clinical significance of congenital vertebral and spinal cord abnormalities. J. Am. Anim. Hosp. Assoc. 11, 426-434.

Balducci, F., Canal, S., Contiero, B. and Bernardini, M. 2017. Prevalence and Risk Factors for Presumptive Ascending/Descending Myelomalacia in Dogs after Thoracolumbar Intervertebral Disk Herniation. J. Vet. Intern. Med. 31, 498-504.

Barone, G., Ziemer, L.S., Shofer, F.S. and Steinberg, S.A. 2002. Risk factors associated with development of seizures after use of iohexol for myelography in dogs: 182 Cases (1998). J. Am. Vet. Med. Assoc. 220, 1499-1502.

Beltran, E., Dennis, R., Doyle, V., de Stefani, A., Holloway, A. and de Risio, L. 2012. Clinical and magnetic resonance imaging features of canine compressive cervical myelopathy with suspected hydrated nucleus pulposus extrusion. J. Small Anim. Pract. 53, 101-107.

Berlanda, M., Zotti, A., Brandazza, G., Poser, H., Calò, P. and Bernardini, M. 2011. Magnetic resonance and computed tomographic features of 4 cases of canine congenital thoracic vertebral anomalies. Can. Vet. J. 52, 1334-1338.

Bertolini, G. and Prokop, M. 2011. Multidetector-row computed tomography: technical basics and preliminary clinical applications in small animals. Vet. J. 189, 15-26.

Bismuth, C., Ferrand, F.X., Millet, M., Buttin, P., Fau, D., Cachon, T., Viguier, E., Escriou, C. and Carozzo, C. 2014. Original surgical treatment of thoracolumbar subarachnoid cysts in six chondrodystrophic dogs. Acta Vet. Scand. 56(1), 32. doi: 10.1186/1751-0147-56-32.

Brisson, B.A. 2010. Intervertebral disc disease in dogs. Vet. Clin. North Am. Small Anim. Pract. 40, 82958.

Carroll, G.L., Keene, B.W. and Forrest, L.J. 1997. Asystole associated with iohexol myelography in a dog. Vet. Radiol. Ultrasound 38, 284-287.

Carstens, A. and Kitshoff, A.M. 2007. Imaging diagnosis--postmyelographic epidural leakage due to increased meningeal permeability. Vet. Radiol. Ultrasound 48, 554-556. 
Cauduro, A., Favole, P., Lorenzo, V., Simonetto, L., Barda, B., Cantile, C. and Asperio, R.M. 2011. Paraparesis caused by vertebral canal leishmaniotic granuloma in a dog. J. Vet. Intern. Med. 25, 398399.

Cherubini, G.B., Platt, S.R., Anderson, T.J., Rusbridge, C., Lorenzo, V., Mantis, P. and Cappello, R. 2006. Characteristics of magnetic resonance images of granulomatous meningoencephalomyelitis in 11 dogs. Vet. Rec. 159, 110-115.

Cooper, J.J., Young, B.D., Griffin, J.F. $4^{\text {th }}$, Fosgate, G.T. and Levine, J.M. 2014. Comparison between noncontrast computed tomography and magnetic resonance imaging for detection and characterization of thoracolumbar myelopathy caused by intervertebral disk herniation in dogs. Vet. Radiol. Ultrasound 55, 182-189.

da Costa, R.C., Parent, J.M. and Dobson, H. 2011. Incidence of and risk factors for seizures after myelography performed with iohexol in dogs: 503 cases (2002-2004). J. Am. Vet. Med. Assoc. 238, 1296-1300.

da Costa, R.C. and Samii, V.F. 2010. Advanced imaging of the spine in small animals. Vet. Clin. North Am. Small Anim. Pract. 40, 765-790.

de Lahunta, A. and Glass, E. 2009. Veterinary Neuroanatomy and Clinical Neurology. $3^{\text {rd }}$ edn, Elsevier, St. Louis, Miss, USA, pp: 65.

Dennis, R. 2011. Optimal magnetic resonance imaging of the spine. Vet. Radiol. Ultrasound 52(1 Suppl. 1), S72-80.

Dennison, S.E., Drees, R., Rylander, H., Yandell, B.S., Milovancev, M., Pettigrew, R. and Schwarz, T. 2010. Evaluation of different computed tomography techniques and myelography for the diagnosis of acute canine myelopathy. Vet. Radiol. Ultrasound 51, 254-258.

De Risio, L., Adams, V., Dennis, R. and McConnell, F.J. 2009. Association of clinical and magnetic resonance imaging findings with outcome in dogs with presumptive acute noncompressive nucleus pulposus extrusion: 42 cases (2000-2007). J. Am. Vet. Med. Assoc. 234, 495-504.

Dolera, M., Malfassi, L., Marcarini, S., Mazza, G., Sala, M., Carrara, N., Facchini, R.V. and Finesso, S. 2015. Hydrated nucleus pulposus extrusion in dogs: correlation of magnetic resonance imaging and microsurgical findings. Acta Vet. Scand. 57, 58.

Favole, P., Cauduro, A., Opreni, M., Zanzani, S., Albonico, F., Manfredi, M., Cantile, C. and Lorenzo, V. 2013. Epidural dirofilariosis in a paraparetic cat: case report of Dirofilaria immitis infection. J. Feline Med. Surg. 15, 1160-1164.

Fisher, M. 2002. Disseminated granulomatous meningoencephalomyelitis in a dog. Can. Vet. J. 43, 49-51.
Funkquist, B. 2008. Thoraco-Lumbar Myelography with Water-Soluble Contrast Medium in Dogs I. Technique of Myelography; Side-effects and Complications. J. Small Anim. Pract. 3, 53-66.

Griffin, J.F.4 ${ }^{\text {th }}$, Levine, J., Kerwin, S. and Cole, R. 2009 Canine thoracolumbar invertebral disk disease: diagnosis, prognosis, and treatment. Compend. Contin. Educ. Vet. 31(3), E3.

Jeffery, N.D. 2010. Vertebral fracture and luxation in small animals. Vet. Clin. North Am. Small Anim. Pract. 40, 809-828.

Jeffery, N.D., Levine, J.M., Olby, N.J. and Stein, V.M. 2013. Intervertebral disk degeneration in dogs: consequences, diagnosis, treatment, and future directions. J. Vet. Intern. Med. 27, 1318-1333.

Hague, D.W., Joslyn, S., Bush, W.W., Glass, E.N. and Durham, A.C. 2015. Clinical, magnetic resonance imaging, and histopathologic findings in $6 \mathrm{dogs}$ with surgically resected extraparenchymal spinal cord hematomas. J. Vet. Intern. Med. 29, 225-230.

Harder, L.K., Ludwig, D.C., Galindo-Zamora, V., Nolte, I. and Wefstaedt, P. 2015. "Disk extension beyond the interspace": an investigation into an alternative nomenclature in diagnostic imaging for displaced canine intervertebral disk material. BMC Vet. Res. 11, 110. doi: 10.1186/s12917-015-0421$\mathrm{X}$.

Hecht, S., Thomas, W.B., Marioni-Henry, K., Echandi, R.L., Matthews, A.R. and Adams, W.H. 2009. Myelography vs computed tomography in the evaluation of acute thoracolumbar intervertebral disk extrusion in chondrodystrophic dogs. Vet. Radiol. Ultrasound 50, 353-359.

Israel, S.K., Levine, J.M., Kerwin, S.C., Levine, G.J. and Fosgate, G.T. 2009. The relative sensitivity of computed tomography and myelography for identification of thoracolumbar intervertebral disk herniations in dogs. Vet. Radiol. Ultrasound 50, 247-252.

Ito, D., Matsunaga, S., Jeffery, N.D., Sasaki, N., Nishimura, R., Mochizuki, M., Kasahara, M., Fujiwara, R. and Ogawa, H. 2005. Prognostic value of magnetic resonance imaging in dogs with paraplegia caused by thoracolumbar intervertebral disk extrusion: 77 cases (2000-2003). J. Am. Vet. Med. Assoc. 227, 1454-1460.

Kamishina, H., Ogawa, H., Katayama, M., Yasuda, J., Sato, R. and Tohyama, K. 2010. Spontaneous regression of a cervical intraspinal cyst in a dog. Vet. Med. Sci. 72, 349-352.

Kirberger, R.M. and Wrigley, R.H. 1993. Myelography in the dog - Review of patients with contrast medium in the central canal. Vet. Radiol. Ultrasound 34, 253-258.

Kishimoto, M., Yamada, K., Ueno, H., Kobayashi, Y. and Wisner, E.R. 2003. Spinal cord effects from 
lumbar myelographic injection technique in the dog. J. Vet. Med. Sci. 66, 67-69.

Konar, M., Lang, J., Flühmann, G. and Forterre, F. 2008. Ventral intraspinal cysts associated with the intervertebral disc: magnetic resonance imaging observations in seven dogs. Vet. Surg. 37, 94-101.

Lorenz, M.D., Coates, J.R. and Kent, M. 2011. Handbook of veterinary neurology. Elsevier Sauders, St Louis.

Manunta, M.L., Evangelisti, M.A., Bergknut, N., Grinwis, G.C., Ballocco, I. and Meij, B.P. 2015. Hydrated nucleus pulposus herniation in seven dogs. Vet. J. 203, 342-344.

Masciarelli, A.E., Griffin, J.F. 4th, Fosgate, G.T., Hecht, S., Mankin, J.M., Holmes, S.P., Platt, S.R., Kent, M., Pancotto, T.E., Chen, A.V. and Levine, J.M. 2017. Evaluation of magnetic resonance imaging for the differentiation of inflammatory, neoplastic, and vascular intradural spinal cord diseases in the dog. Vet. Radiol. Ultrasound 58, 444-453.

Mauler, D.A., De Decker, S., De Risio, L., Volk, H.A., Dennis, R., Gielen, I., Van der Vekens, E., Goethals, K. and Van Ham, L. 2017. Spinal Arachnoid Diverticula: Outcome in 96 Medically or Surgically Treated Dogs. J. Vet. Intern. Med. 31, 849-853.

McDonnell, J.J., Knowles, K.E., deLahunta, A., Bell, J.S., Lowrie, C.T. and Todhunter, R.J. 2003. Thoracolumbar spinal cord compression due to vertebral process degenerative joint disease in a family of Shiloh Shepherd dogs. J. Vet. Intern. Med. 17, 530-537.

Morgan, J.P. 1999. Transitional lumbosacral vertebral anomaly in the dog: A radiographic study. J. Small Anim. Pract. 40, 167-172.

Murata, D., Miura, N., Iwanaga, T., Tokunaga, S., Miyoshi, N., Kitano, Y., Momoi, Y. and Fujiki, M. 2012. CT and MRI Imaging Diagnosis of Epidural Idiopathic Sterile Pyogranulomatous Inflammation in a Dog Spinal Canal. J. Vet. Med. Sci. 74, 913915.

Newitt, A., German, A.J. and Barr, F.J. 2008. Congenital abnormalities of the feline vertebral column. Vet. Radiol. Ultrasound 49, 35-41.

Nykamp, S.G., Steffey, M.A., Scrivani, P.V. and Schatzberg, S.J. 2003. Computed tomographic appearance of epidural empyema in a dog. Can. Vet. J. 44, 729-731.

Olby, N.J., Munana, K.R., Sharp, N.J. and Thrall, D.E. 2000. The computed tomographic appearance of acute thoracolumbar intervertebral disc herniations in dogs. Vet. Radiol. Ultrasound 41, 396-402.

Packer, R.A., Bergman, R.L., Coates, J.R., Essman, S.C., Weis, K., O'Brien, D.P. and Johnson, G.C. 2007. Intracranial subarachnoid hemorrhage following lumbar myelography in two dogs. Vet. Radiol. Ultrasound 48, 323-327.

Paithanpagare, Y.M., Tank, P.H., Mankad, M.Y., Shirodkar, K. and Derashri, H.J. 2008. Myelography in dogs. Vet. World 1, 152-154.

Parker, A.J., Cusick, P.K., Stowater, J.L. and Park, R.D. 1975. Effects of intramedullary injection of methiodal and lidocaine on spinal cords of dogs. Am. J. Vet. Res. 36, 1529-1532.

Penderis, J., Sullivan, M., Schwarz, T. and Griffiths, I.R. 1999. Subdural injection of contrast medium as a complication of myelography. J. Small Anim. Pract. 40, 173-176.

Platt, S. and Garosi, L. 2012. Small animals neurological emergencies. London, Manson Publishing Press.

Reginato, A., Giannuzzi, P., Ricciardi, M., De Simone, A., Sanguinetti, M., Porcellato, I., Mandara, M.T. 2014. Extradural spinal cord lesion in a dog: first case study of canine neurological histoplasmosis in Italy. Vet. Microbiol. 170, 451-455.

Ricciardi, M. 2016. Usefulness of multidetector computed tomography in the evaluation of spinal neuro-musculoskeletal injuries. Vet. Comp. Orthop. Traumatol. 29, 1-13.

Ricciardi, M. 2018. Principles and applications of the balanced steady-state free precession sequence in small animal low-field MRI. Vet. Res. Commun. $42,65-86$.

Robertson, I. and Thrall, D.E. 2011. Imaging dogs with suspected disc herniation: pros and cons of myelography, computed tomography, and magnetic resonance. Vet. Radiol. Ultrasound 52, S81-84.

Royaux, E., Martlé, V., Kromhout, K., Van der Vekens, E., Broeckx, B.J., Van Ham, L. and Gielen, I. 2016. Detection of compressive hydrated nucleus pulposus extrusion in dogs with multislice computed tomography. Vet. J. 216, 202-206.

Seiler, G.S., Robertson, I.D., Mai, W., Widmer, W.R., Suran, J., Nemanic, S., Lamb, C.R., Lang, J., Johnson, J.L. and Thrall, D.E. 2012. Usefulness of a half-fourier acquisition single-shot turbo spinecho pulse sequence in identifying arachnoid diverticula in dogs. Vet. Radiol. Ultrasound 53, 157-161.

Shores, A. and Brisson, B.A. 2017. Current Techniques in Canine and Feline Neurosurgery. Hoboken NJ (USA), Wiley-Blackwell, pp: 81.

Tator, C.H. and Fehlings, M.G. 1991. Review of the secondary injury theory of acute spinal cord trauma with emphasis on vascular mechanisms. J. Neurosurg. 75, 15-26.

Thornbury, J.R., Fryback, D.G., Turski, P.A., Javid, M.J., McDonald, J.V., Beinlich, B.R., Gentry, L.R., Sackett, J.F., Dasbach, E.J. and Martin, P.A. 1993. Disk-caused nerve compression in patients with 
acute low-back pain: diagnosis with MR, CT myelography, and plain CT. Radiology 186, 731738.

Wessmann, A., Lu, D., Lamb, C.R., Smyth, B., Mantis, P., Chandler, K., Boag, A., Cherubini, G.B. and Cappello, R. 2006. Brain and spinal cord haemorrhages associated with Angiostrongylus vasorum infection in four dogs. Vet. Rec. 158, 858863.

Wisner, E. and Zwingenberger, A. 2015. Atlas of Small Animal CT and MRI. 2015. Oxford, WileyBlackwell, pp: 295. 